TRANSACTIONS OF THE

AMERICAN MATHEMATICAL SOCIETY

Volume 353, Number 8, Pages 3181-3191

S 0002-9947(01)02747-7

Article electronically published on April 12, 2001

\title{
CLASSIFYING SUBCATEGORIES OF MODULES
}

\author{
MARK HOVEY
}

\begin{abstract}
Let $R$ be the quotient of a regular coherent commutative ring by a finitely generated ideal. In this paper, we classify all abelian subcategories of finitely presented $R$-modules that are closed under extensions. We also classify abelian subcategories of arbitrary $R$-modules that are closed under extensions and coproducts, when $R$ is commutative and Noetherian. The method relies on comparison with the derived category of $R$.
\end{abstract}

\section{INTRODUCTION}

A basic problem in mathematics is to classify all objects one is studying up to isomorphism. A lesson this author learned from stable homotopy theory [HS98] is that while this is almost always impossible, it is sometimes possible, and very useful, to classify certain full subcategories of the category one is working in. In particular, if $R$ is a commutative ring, the thick subcategories of small objects in the derived category $\mathcal{D}(R)$ have been classified. Recall that a thick subcategory is a triangulated subcategory closed under summands. Thick subcategories correspond to unions of subsets of Spec $R$ of the form $V(\mathfrak{a})$, where $\mathfrak{a}$ is a finitely generated ideal of $R$. In particular, when $R$ is Noetherian, they correspond to arbitrary unions of closed sets of Spec $R$. This line of research was initiated by Hopkins [Hop87], whose flawed proof of the classification was corrected by Neeman [Nee92] in the Noetherian case. Thomason Tho97] generalized the result to arbitrary commutative rings (and to quasi-compact, quasi-separated schemes).

The author has long thought that the analogous classification in the ostensibly simpler category of $R$-modules is the classification of torsion theories when $R$ is a Noetherian and commutative ring [Ste75, Section VI.6]. After all, these too correspond to arbitrary unions of closed sets in $\operatorname{Spec} R$. However, we show in this paper that the analog of a thick subcategory in $\mathcal{D}(R)$ is not a torsion theory of $R$-modules, but just an Abelian subcategory of $R$-modules closed under extensions. We call this a wide subcategory. We use the classification of thick subcategories mentioned above to give a classification of wide subcategories of finitely presented modules over a large class of commutative coherent rings. To be precise, our classification works for quotients of regular commutative coherent rings by finitely generated ideals. Recall that a coherent ring is regular if every finitely generated ideal has finite projective dimension. Thus our classification includes, for example, the polynomial ring on countably many variables over a principal ideal domain, and all finitely generated algebras over a field. A corollary of our result is that if $R$ is Noetherian as well, then every wide subcategory of finitely presented $R$-modules is

Received by the editors January 15, 2000 and, in revised form, June 19, 2000.

2000 Mathematics Subject Classification. Primary 13C05, 18E30, 18G35.

(C)2001 American Mathematical Society 
in fact the collection of all finitely presented modules in a torsion theory; that is, an Abelian subcategory of finitely presented $R$-modules that is closed under extensions is in fact closed under arbitrary submodules and quotient modules. It is interesting that we have no direct proof of this fact, but must resort to the rather difficult classification of thick subcategories in the derived category.

One can also classify thick subcategories closed under arbitrary coproducts, or arbitrary products. These are called localizing and colocalizing subcategories, respectively. For $\mathcal{D}(R)$ when $R$ is a Noetherian commutative ring, they were classified by Neeman [Nee92], and correspond to arbitrary subsets of $\operatorname{Spec} R$. We give an analogous classification of wide subcategories closed under arbitrary coproducts in the Noetherian case. The proof again relies on comparison with the derived category.

\section{Wide SUBCATEGORIES}

Suppose $R$ is a ring. In this section, we define wide subcategories of $R$-modules and construct an adjunction to thick subcategories of $\mathcal{D}(R)$. The natural domain of this adjunction is actually $\mathcal{L}_{\text {wide }}(R)$, the lattice of wide subcategories of $\mathcal{C}_{0}$, the wide subcategory generated by $R$. When $R$ is coherent, we identify $\mathcal{C}_{0}$ with the finitely presented modules, but we do not know what it is in general.

We recall that a thick subcategory of a triangulated category like $\mathcal{D}(R)$ is a full triangulated subcategory closed under retracts (summands). This means, in particular, that if we have an exact triangle $X \rightarrow Y \rightarrow Z \rightarrow \Sigma X$ and two out of three of $X, Y$, and $Z$ are in the thick subcategory, so is the third.

The analogous definition for subcategories of an Abelian category is the following.

Definition 1.1. A full subcategory $\mathcal{C}$ of $R$-mod, or any Abelian category, is called wide if it is Abelian and closed under extensions.

When we say a full subcategory $\mathcal{C}$ is Abelian, we mean that if $f: M \rightarrow N$ is a map of $\mathcal{C}$, then the kernel and cokernel of $f$ are in $\mathcal{C}$. Thus a wide subcategory $\mathcal{C}$ need not be closed under arbitrary subobjects or quotient objects. However, $\mathcal{C}$ is automatically closed under summands. Indeed, if $M \cong N \oplus P$ and $M \in \mathcal{C}$, then $N$ is the kernel of the self-map of $M$ that takes $(n, p)$ to $(0, p)$. Thus $N \in \mathcal{C}$. In particular, $\mathcal{C}$ is replete, in the sense that anything isomorphic to something in $\mathcal{C}$ is itself in $\mathcal{C}$.

Torsion theories and Serre classes are closely related to wide subcategories. Recall that a Serre class is just a wide subcategory closed under arbitrary subobjects, and hence arbitrary quotient objects. Similarly, a (hereditary) torsion theory is a Serre class closed under arbitrary direct sums. In particular, the empty subcategory, the 0 subcategory, and the entire category of $R$-modules are torsion theories, and so wide subcategories. The category of all $R$-modules of cardinality $\leq \kappa$ for some infinite cardinal $\kappa$ is a Serre class (but not a torsion theory), and hence a wide subcategory. The category of finite-dimensional rational vector spaces, as a subcategory of the category of abelian groups, is an example of a wide subcategory that is not a Serre class. The thick subcategories studied in [HP99, HP00] are, on the other hand, more general than wide subcategories.

Note that the collection of all wide subcategories of $R$-mod forms a complete lattice (though it is not a set). Indeed, the join of a collection of wide subcategories is the wide subcategory generated by them all, and the meet of a collection of wide subcategories is their intersection. 
The following proposition shows that wide subcategories are the analogue of thick subcategories.

Proposition 1.2. Suppose $\mathcal{C}$ is a wide subcategory of $R$-mod. Define $f(\mathcal{C})$ to be the collection of all small objects $X \in \mathcal{D}(R)$ such that $H_{n} X \in \mathcal{C}$ for all $n \in \mathbb{Z}$. Then $f(\mathcal{C})$ is a thick subcategory.

Recall that an object $X$ in a triangulated category $\mathcal{T}$ is small when $\mathcal{T}(X,-)$ preserves coproducts. A chain complex $X$ is small in $\mathcal{D}(R)$ if and only if $X$ is quasi-isomorphic to a finite complex of finitely generated projectives.

Note that the collection of all thick subcategories is also a complete lattice, and the map $f$ is clearly order-preserving.

Proof. Since wide subcategories are closed under summands, $f(\mathcal{C})$ is closed under retracts. It is clear that $X \in f(\mathcal{C})$ if and only if $\Sigma X \in f(\mathcal{C})$. It remains to show that, if we have an exact triangle $X \rightarrow Y \rightarrow Z \rightarrow \Sigma X$ and $X, Z \in f(\mathcal{C})$, then $Y \in f(\mathcal{C})$. We have a short exact sequence

$$
0 \rightarrow A \rightarrow H_{n} Y \rightarrow B \rightarrow 0
$$

where $A$ is the cokernel of the map $H_{n+1} Z \rightarrow H_{n} X$, and $B$ is the kernel of the map $H_{n} Z \rightarrow H_{n-1} X$. Hence $A$ and $B$ are in $\mathcal{C}$, and so $H_{n} Y \in \mathcal{C}$ as well. Thus $Y \in f(\mathcal{C})$.

Note that Proposition 1.2 remains true if $R$-mod is replaced by any Abelian category, or indeed, if $\mathcal{D}(R)$ is replaced by a stable homotopy category [HPS97] and $R$ is replaced by the homotopy of the sphere in that category.

Proposition 1.2 implies that the homology of a small object in $\mathcal{D}(R)$ must lie in the wide subcategory generated by $R$.

Corollary 1.3. Let $\mathcal{C}_{0}$ be the wide subcategory generated by $R$. If $X$ is a small object of $\mathcal{D}(R)$, then $H_{n} X \in \mathcal{C}_{0}$ for all $n$ and $H_{n} X=0$ for all but finitely many $n$.

Proof. By hypothesis, the complex $S^{0}$ consisting of $R$ concentrated in degree 0 is in $f\left(\mathcal{C}_{0}\right)$. Therefore, the thick subcategory $\mathcal{D}$ generated by $S^{0}$ is contained in $f\left(\mathcal{C}_{0}\right)$. But $\mathcal{D}$ is precisely the small objects in $\mathcal{D}(R)$. (This is proved in HPS97. Corollary 2.3.12] for commutative $R$, but the proof does not require commutativity.) Hence $H_{n} X \in \mathcal{C}_{0}$ for all small objects $X$ and all $n$. It remains to prove that $H_{n} X=0$ for all but finitely many $n$ if $X$ is small. This is proved analogously; the collection of all such $X$ is a thick subcategory containing $S^{0}$.

This corollary tells us that the proper domain of $f$ is $\mathcal{L}_{\text {wide }}(R)$, the lattice of wide subcategories of $\mathcal{C}_{0}$. We would like $f$ to define an isomorphism $f: \mathcal{L}_{\text {wide }}(R) \rightarrow$ $\mathcal{L}_{\text {thick }}(\mathcal{D}(R))$, where $\mathcal{L}_{\text {thick }}(\mathcal{D}(R))$ is the lattice of thick subcategories of small objects in $\mathcal{D}(R)$. We now construct the only possible inverse to $f$.

Given a thick subcategory $\mathcal{D} \in \mathcal{L}_{\text {thick }}(\mathcal{D}(R))$, we define $g(\mathcal{D})$ to be the wide subcategory generated by $\left\{H_{n} X\right\}$, where $X$ runs though objects of $\mathcal{D}$ and $n$ runs through $\mathbb{Z}$. By Corollary $1.3, g(\mathcal{D}) \in \mathcal{L}_{\text {wide }}(R)$. Also, $g$ is obviously orderpreserving. We also point out that, like $f, g$ can be defined in considerably greater generality.

We then have the following proposition.

Proposition 1.4. The lattice homomorphism $g$ is left adjoint to $f$. That is, for $\mathcal{C} \in \mathcal{L}_{\text {wide }}(R)$ and $\mathcal{D} \in \mathcal{L}_{\text {thick }}(\mathcal{D}(R))$, we have $g(\mathcal{D}) \subseteq \mathcal{C}$ if and only if $\mathcal{D} \subseteq f(\mathcal{C})$. 
Proof. Suppose first that $g(\mathcal{D}) \subseteq \mathcal{C}$. This means that for every $X \in \mathcal{D}$, we have $H_{n} X \in \mathcal{C}$ for all $n$. Hence $X \in f(\mathcal{C})$. Thus $\mathcal{D} \subseteq f(\mathcal{C})$. Conversely, if $\mathcal{D} \subseteq f(\mathcal{C})$, then for every $X \in \mathcal{D}$ we have $H_{n} X \in \mathcal{C}$ for all $n$. Thus $g(\mathcal{D}) \subseteq \mathcal{C}$.

Corollary 1.5. Suppose $R$ is a ring. If $\mathcal{C} \in \mathcal{L}_{\text {wide }}(R)$, then $g f(\mathcal{C})$ is the smallest wide subcategory $\mathcal{C}^{\prime}$ such that $f\left(\mathcal{C}^{\prime}\right)=f(\mathcal{C})$. Similarly, if $\mathcal{D} \in \mathcal{L}_{\text {thick }}(\mathcal{D}(R))$, then $f g(\mathcal{D})$ is the largest thick subcategory $\mathcal{D}^{\prime}$ such that $g\left(\mathcal{D}^{\prime}\right)=g(\mathcal{D})$.

Proof. This corollary is true for any adjunction between partially ordered sets. For example, if $f\left(\mathcal{C}^{\prime}\right)=f(\mathcal{C})$, then $g f\left(\mathcal{C}^{\prime}\right)=g f(\mathcal{C})$. But $g f\left(\mathcal{C}^{\prime}\right) \subseteq \mathcal{C}^{\prime}$, so $g f(\mathcal{C}) \subseteq \mathcal{C}^{\prime}$. Furthermore, combining the counit and unit of the adjunction shows that $\operatorname{fg} f(\mathcal{C})$ is contained in and contains $f(\mathcal{C})$. The other half is similar.

It follows from this corollary that $f$ is injective if and only $g f(\mathcal{C})=\mathcal{C}$ for all $\mathcal{C} \in$ $\mathcal{L}_{\text {wide }}(R)$ and that $f$ is surjective if and only if $f g(\mathcal{D})=\mathcal{D}$ for all $\mathcal{D} \in \mathcal{L}_{\text {thick }}(\mathcal{D}(R))$.

In order to investigate these questions, it would be a great help to understand $\mathcal{C}_{0}$, the wide subcategory generated by $R$. We know very little about this in general, except that $\mathcal{C}_{0}$ obviously contains all finitely presented modules and all finitely generated ideals of $R$. We also point out that $\mathcal{C}_{0}$ is contained in the wide subcategory consisting of all modules of cardinality $\leq \kappa$, where $\kappa$ is the larger of $\omega$ and the cardinality of $R$. In particular, $\mathcal{C}_{0}$ has a small skeleton, and so there is only a set of wide subcategories of $\mathcal{C}_{0}$.

The only case where we can identify $\mathcal{C}_{0}$ is when $R$ is a coherent ring. A brief description of coherent rings can be found in Ste75. Section I.13]; an excellent reference for deeper study is Gla89.

Lemma 1.6. A ring $R$ is coherent if and only if the wide subcategory $\mathcal{C}_{0}$ generated by $R$ consists of the finitely presented modules.

Proof. Suppose first that $\mathcal{C}_{0}$ is the collection of finitely presented modules. Suppose $\mathfrak{a}$ is a finitely generated left ideal of $R$. Then $R / \mathfrak{a}$ is a finitely presented module, so $\mathfrak{a}$, as the kernel of the map $R \rightarrow R / \mathfrak{a}$, is in $\mathcal{C}_{0}$. Hence $\mathfrak{a}$ is finitely presented, and so $R$ is coherent.

The collection of finitely presented modules over any ring is clearly closed under cokernels and is also closed under extensions. Indeed, suppose we have a short exact sequence

$$
0 \rightarrow M^{\prime} \rightarrow M \rightarrow M^{\prime \prime} \rightarrow 0
$$

where $M^{\prime}$ and $M^{\prime \prime}$ are finitely presented. Choose a finitely generated projective $P$ and a surjection $P \rightarrow M^{\prime \prime}$. We can lift this to a map $P \rightarrow M$. Then we get a surjection $M^{\prime} \oplus P \rightarrow M$, as is well-known. Furthermore, the kernel of this surjection is the same as the kernel of $P \rightarrow M^{\prime \prime}$, which is finitely generated since $M^{\prime \prime}$ is finitely presented. Hence $M$ is the quotient of the finitely presented module $M^{\prime} \oplus P$ by a finitely generated submodule, so is finitely presented.

Now suppose that $R$ is coherent. We show that the kernel of a map $f: M \rightarrow N$ of finitely presented modules is finitely presented. The point is that the image of $f$ is a finitely generated submodule of the finitely presented module $N$. Because the ring is coherent, this means that the image of $f$ is finitely presented. The kernel of $f$ is therefore finitely generated, but it is a submodule of the finitely presented module $M$, so it is finitely presented, using coherence again. 
Noetherian rings can be characterized in a similar manner as rings in which $\mathcal{C}_{0}$ is the collection of finitely generated modules.

\section{Surjectivity of the Adjunction}

The goal of this section is to show that the map $f: \mathcal{L}_{\text {wide }}(R) \rightarrow \mathcal{L}_{\text {thick }}(\mathcal{D}(R))$ is surjective for all commutative rings $R$. This is a corollary of Thomason's classification of thick subcategories in $\mathcal{D}(R)$.

Suppose $R$ is a commutative ring. Denote by $J(\operatorname{Spec} R) \subseteq 2^{\operatorname{Spec} R}$ the collection of order ideals in $\operatorname{Spec} R$, so that $S \in J(\operatorname{Spec} R)$ if and only if $\mathfrak{p} \in S$ and $\mathfrak{q} \subseteq \mathfrak{p}$ implies that $\mathfrak{q} \in S$. Note that an open set in the Zariski topology of $\operatorname{Spec} R$ is in $J(\operatorname{Spec} R)$, so an arbitrary intersection of open sets is in $J(\operatorname{Spec} R)$. Also, note that $J(\operatorname{Spec} R)$ is a complete distributive lattice.

We will construct a chain of maps

$$
J(\operatorname{Spec} R)^{\text {op }} \stackrel{i}{\rightarrow} \mathcal{L}_{\text {tors }}(R) \stackrel{j}{\rightarrow} \mathcal{L}_{\text {Serre }}(R) \stackrel{\alpha}{\longrightarrow} \mathcal{L}_{\text {wide }}(R) \stackrel{f}{\rightarrow} \mathcal{L}_{\text {thick }}(\mathcal{D}(R)),
$$

each of which is a right adjoint. We have of course already constructed $f$.

To construct $i$, note that $\mathcal{L}_{\text {tors }}(R)$ denotes the lattice of all torsion theories of $R$ modules. Recall that a torsion theory is a wide subcategory closed under arbitrary submodules and arbitrary direct sums. The map $i$ is defined by

$$
i(S)=\left\{M \mid M_{(\mathfrak{p})}=0 \text { for all } \mathfrak{p} \in S\right\} .
$$

Its left adjoint $r$ has

$$
r(\mathcal{T})=\left\{\mathfrak{p} \mid M_{(\mathfrak{p})}=0 \text { for all } M \in \mathcal{T}\right\}=\bigcap_{M \in \mathcal{T}}(\operatorname{Spec} R \backslash \operatorname{supp} M) .
$$

One can check that $\operatorname{ri}(S)=S$, so that $i$ is an embedding. In case $R$ is a Noetherian commutative ring, $i$ is an isomorphism Ste75, Section VI.6].

To construct $j$, let $\mathcal{S}_{0}$ denote the Serre class generated by $R$. Recall that a Serre class is a wide subcategory closed under arbitrary subobjects. If $R$ is Noetherian, then $\mathcal{S}_{0}$ is the finitely generated $R$-modules, but in general it will be larger than this. The symbol $\mathcal{L}_{\text {Serre }}(R)$ denotes the lattice of Serre subclasses of $\mathcal{S}_{0}$. The map $j$ takes a torsion theory $\mathcal{T}$ to its intersection with $\mathcal{S}_{0}$. Its left adjoint $s$ takes a Serre subclass of $\mathcal{S}_{0}$ to the torsion theory it generates. Since a torsion theory is determined by the finitely generated modules in it (since it is closed under direct limits), the composite $s j$ is the identity. Thus $j$ is also an embedding, for an arbitrary ring $R$. When $R$ is Noetherian, $j$ is an isomorphism. Indeed, in this case, the collection of modules all of whose finitely generated submodules lie in a Serre subclass $\mathcal{S}$ of finitely generated modules is a torsion theory, and is therefore $s(\mathcal{S})$. Hence $j s$ is the identity as well.

The map $\alpha$ takes a Serre class to its intersection with $\mathcal{C}_{0}$. Its adjoint $\beta$ takes a wide subcategory to the Serre class it generates. When $R$ is Noetherian, $\mathcal{C}_{0}$ and $\mathcal{S}_{0}$ coincide, so one can easily see that $\beta \alpha$ is the identity, so that $\alpha$ is injective. However, $\alpha$ will not be injective in general, as we will see at the end of this section.

Note that the composite $f \alpha j i$ takes $S \in J(\operatorname{Spec} R)$ to the collection of all small objects $X$ in $\mathcal{D}(R)$ such that $\left(H_{n} X\right)_{(\mathfrak{p})}=0$ for all $\mathfrak{p} \in S$ and all $n$. Since $H_{n}\left(X_{(\mathfrak{p})}\right) \cong$ $\left(H_{n} X\right)_{(\mathfrak{p})}$, this is the same as the collection of all small $X$ such that $X_{(\mathfrak{p})}=0$ for all $\mathfrak{p} \in S$.

The following theorem is the main result of [Tho97. To describe it, recall that the open subsets of the Zariski topology on $\operatorname{Spec} R$, where $R$ is commutative, are 
the sets $D(\mathfrak{a})$ where $\mathfrak{a}$ is an ideal of $R$ and $D(\mathfrak{a})$ consists of all primes that do not contain $\mathfrak{a}$. The open set $D(\mathfrak{a})$ is quasi-compact if and only if $D(\mathfrak{a})=D(\mathfrak{b})$ for some finitely generated ideal $\mathfrak{b}$ of $R$. This fact is well-known in algebraic geometry, and can be deduced from the argument on the top of p. 72 in [Har77. Now we let $\widetilde{J}(\operatorname{Spec} R)$ denote the sublattice of $J(\operatorname{Spec} R)$ consisting of arbitrary intersections of quasi-compact open sets.

Theorem 2.1 (Thomason's theorem). Let $R$ be a commutative ring. Let $h$ denote the restriction of faji to $\widetilde{J}(\operatorname{Spec} R)$. Then $h: \widetilde{J}(\operatorname{Spec} R)^{\text {op }} \rightarrow \mathcal{L}_{\text {thick }}(\mathcal{D}(R))$ is an isomorphism.

The following corollary is immediate, since $f \alpha j i$ is surjective.

Corollary 2.2. Let $R$ be a commutative ring. Then the map $f: \mathcal{L}_{\text {wide }}(R) \rightarrow$ $\mathcal{L}_{\text {thick }}(\mathcal{D}(R))$ is surjective. In particular, for any thick subcategory $\mathcal{D}$, we have $f g(\mathcal{D})=\mathcal{D}$.

Note that Thomason's theorem implies that $f \alpha j i$ is not injective whenever $\widetilde{J}(\operatorname{Spec} R) \neq J(\operatorname{Spec} R)$. Since $i$ and $j$ are injective, one of $f$ or $\alpha$ must not be injective in this case. The goal of this paper is to show that $f$ is an isomorphism whenever $R$ is the quotient of a regular coherent ring by a finitely generated ideal. Therefore, $\alpha$ will not be injective in general. For example, when $R$ is isomorphic to a polynomial ring on infinitely many variables $x_{i}$ over a field $k$, the Serre class generated by $k$ gets sent to the zero wide subcategory by $\alpha$. This means that torsion theories and Serre classes cannot classify thick subcategories of $\mathcal{D}(R)$ in general, though they do in the Noetherian case.

\section{Regular COHEREnt RingS}

The goal of this section is to show that the map $f: \mathcal{L}_{\text {wide }}(R) \rightarrow \mathcal{L}_{\text {thick }}(\mathcal{D}(R))$ is an isomorphism when $R$ is a regular coherent commutative ring. Regularity means that every finitely presented module has finite projective dimension; see Gla89. Section 6.2] for many results about regular coherent rings. An example of a regular coherent ring that is not Noetherian is the polynomial ring on infinitely many variables over a principal ideal domain.

As we have already seen, $f$ is injective if and only $g f(\mathcal{C})=\mathcal{C}$ for all wide subcategories of finitely presented modules (when $R$ is coherent). We start out by proving that $g f\left(\mathcal{C}_{0}\right)=\mathcal{C}_{0}$ when $R$ is coherent.

Proposition 3.1. Suppose $R$ is a ring, and $M$ is a finitely presented $R$-module. Then there is a small object $X \in \mathcal{D}(R)$ such that $H_{0} X \cong M$.

Proof. Write $M$ as the cokernel of a map $f: R^{m} \rightarrow R^{n}$. Recall that, given a module $N, S^{0} N$ denotes the complex that is $N$ concentrated in degree 0 . Define $X$ to be the cofiber of the induced map $S^{0} R^{m} \rightarrow S^{0} R^{n}$. Then $X$ is small and $H_{0} X=M\left(\right.$ and $H_{1} X$ is the kernel of $f$ ).

Corollary 3.2. Suppose $R$ is a coherent ring, and $\mathcal{C}_{0}$ is the subcategory of finitely presented modules. Then $g f\left(\mathcal{C}_{0}\right)=\mathcal{C}_{0}$.

In order to prove that $g f(\mathcal{C})=\mathcal{C}$ in general, however, given a finitely presented module $M$, we would have to find a complex $X$ that is small in $\mathcal{D}(R)$ such that $H_{0} X \cong M$ and each $H_{n} X$ is in the wide subcategory generated by $M$. The obvious 
choice is $S^{0} M$, the complex consisting of $M$ concentrated in degree 0. However, $S^{0} M$ cannot be small in $\mathcal{D}(R)$ unless $M$ has finite projective dimension, as we show in the following lemma. This lemma and the proposition that follows are very similar to [CKN99, Lemma 1.1].

Lemma 3.3. Suppose $R$ is a ring and $M$ is an $R$-module. If the complex $S^{0} M$ is small in $\mathcal{D}(R)$, then $M$ has finite projective dimension.

Proof. Define an object $X$ of $\mathcal{D}(R)$ to have finite projective dimension if there is an $i$ such that $H_{j} F\left(X, S^{0} N\right)=0$ for all $R$-modules $N$ and all $j$ with $|j|>i$. Here $F\left(X, S^{0} N\right)$ is the function complex $\operatorname{Hom}_{R}(Q X, N)$ in $\mathcal{D}(\mathbb{Z})$ obtained by replacing $X$ by a cofibrant chain complex $Q X$ quasi-isomorphic to it. (In the terminology of [Hov98, Chapter 4], the model category $\mathrm{Ch}(R)$ of chain complexes over $R$ with the projective model structure is a $\mathrm{Ch}(\mathbb{Z})$-model category, and we are using that structure.) If $X=S^{0} M$, then $H_{i} F\left(S^{0} M, S^{0} N\right)=\operatorname{Ext}^{-i}(M, N)$, so $S^{0} M$ has finite projective dimension if and only if $M$ does. It is easy to see that complexes with finite projective dimension form a thick subcategory containing $R$. Therefore every small object of $\mathcal{D}(R)$ has finite projective dimension.

Conversely, we have the following proposition.

Proposition 3.4. Suppose $R$ is a coherent ring and $M$ is a finitely presented module of finite projective dimension. Then $S^{0} M$ is small in $\mathcal{D}(R)$.

Proof. It may be possible to give a direct proof of this, but we prefer to use model categories. Theorem 7.4.3 of [Hov98] asserts that any cofibrant complex $A$ that is small in the category $\mathrm{Ch}(R)$ of chain complexes and chain maps, in the sense that $\mathrm{Ch}(R)(A,-)$ commutes with direct limits, will be small in $\mathcal{D}(R)$. Of course, $S^{0} M$ is small in $\operatorname{Ch}(R)$, but it will not be cofibrant. To make it cofibrant, we need to replace $M$ by a projective resolution. Since $M$ is finitely presented and the ring $R$ is coherent, each term $P_{i}$ in a projective resolution for $M$ will be finitely generated. Since $M$ has finite projective dimension, the resolution $P_{*}$ is finite. Hence $P_{*}$ is small in $\mathrm{Ch}(R)$, and so also in $\mathcal{D}(R)$. Since $P_{*}$ is isomorphic to $S^{0} M$ in $\mathcal{D}(R)$, the result follows.

This proposition leads immediately to the following theorem.

Theorem 3.5. Suppose $R$ is a regular coherent ring, and $\mathcal{C}$ is a wide subcategory of finitely presented $R$-modules. Then $g f(\mathcal{C})=\mathcal{C}$.

Proof. We have already seen that $g f(\mathcal{C}) \subseteq \mathcal{C}$. Suppose $M \in \mathcal{C}$. Then $S^{0} M$ is small by Proposition 3.4. so clearly $S^{0} M \in f(\mathcal{C})$. Thus $M \in g f(\mathcal{C})$.

The author believes that this theorem should hold without the regularity hypothesis, although obviously a more clever proof is required. Theorem 3.5 and Corollary 2.2 lead immediately to the following classification theorem.

Theorem 3.6. Suppose $R$ is a regular commutative coherent ring. Then the map $f: \mathcal{L}_{\text {wide }}(R) \rightarrow \mathcal{L}_{\text {thick }}(\mathcal{D}(R))$ is an isomorphism. Hence the restriction of aji defines an isomorphism $\widetilde{h}: \widetilde{J}(\operatorname{Spec} R) \rightarrow \mathcal{L}_{\text {wide }}(R)$ as well.

Corollary 3.7. Suppose $R$ is a regular commutative coherent ring, $\mathcal{C}$ is a wide subcategory of finitely presented $R$-modules, and $M \in \mathcal{C}$. If $N$ is a finitely presented submodule or quotient module of $M$, then $N \in \mathcal{C}$. In particular, if $R$ is also Noetherian, every wide subcategory of finitely generated modules is a Serre class. 
Indeed, the first statement is obviously true for any wide subcategory coming from $\widetilde{J}(\operatorname{Spec} R)$.

\section{Quotients of REgular COHERENT RINGS}

The goal of this section is to understand the relationship between wide subcategories of $R$-modules and wide subcategories of $R / \mathfrak{a}$-modules, where $\mathfrak{a}$ is a two-sided ideal of $R$. This will allow us to extend Theorem 3.6 to quotients of regular commutative coherent rings by finitely generated ideals.

Given $\mathcal{C} \in \mathcal{L}_{\text {wide }}(R / \mathfrak{a})$, we can think of $\mathcal{C}$ as a full subcategory of $R$-modules where $\mathfrak{a}$ happens to act trivially. As such, it will be closed under kernels and cokernels, but not extensions. Define $u(\mathcal{C})$ to be the wide subcategory of $R$-modules generated by $\mathcal{C}$. In order to be sure that $u(\mathcal{C})$ is contained in $\mathcal{C}_{0}(R)$, we need to make sure that $R / \mathfrak{a} \in \mathcal{C}_{0}(R)$. The easiest way to be certain of this is if $\mathfrak{a}$ is finitely generated as a left ideal; under this assumption, we have just defined a map $u: \mathcal{L}_{\text {wide }}(R / \mathfrak{a}) \rightarrow \mathcal{L}_{\text {wide }}(R)$.

As usual, this map has a left adjoint $v$. Given $\mathcal{D} \in \mathcal{L}_{\text {wide }}(R)$, we define $v(\mathcal{D})$ to be the collection of all $M \in \mathcal{D}$ such that $\mathfrak{a}$ acts trivially on $M$, so that $M$ is naturally an $R / \mathfrak{a}$-module. Then $v(\mathcal{D})$ is a wide subcategory. It is not clear that $v(\mathcal{D}) \subseteq \mathcal{C}_{0}(R / \mathfrak{a})$ in general. However, if $R$ is coherent, then any $M$ in $g(\mathcal{D})$ will be finitely presented as an $R$-module, and so finitely presented as an $R / \mathfrak{a}$-module.

Altogether then, we have the following lemma, whose proof we leave to the reader.

Lemma 4.1. Suppose $R$ is a coherent ring and $\mathfrak{a}$ is a two-sided ideal of $R$ that is finitely generated as a left ideal. Then the map $u: \mathcal{L}_{\text {wide }}(R / \mathfrak{a}) \rightarrow \mathcal{L}_{\text {wide }}(R)$ constructed above is right adjoint to the map $v$ constructed above.

We claim that $v u(\mathcal{C})=\mathcal{C}$ for all $\mathcal{C} \in \mathcal{L}_{\text {wide }}(R / \mathfrak{a})$, so that $u$ is in fact an embedding. To see this, we need a description of $u(\mathcal{C})$, or, more generally, a description of the wide subcategory generated by a full subcategory $\mathcal{D}$ that is already closed under kernels and cokernels. It is clear that this wide subcategory will have to contain all extensions of $\mathcal{D}$, so let $\mathcal{D}_{1}$ denote the full subcategory consisting of all extensions of $\mathcal{D}$. An object $M$ is in $\mathcal{D}_{1}$ if and only if there is a short exact sequence

$$
0 \rightarrow M^{\prime} \rightarrow M \rightarrow M^{\prime \prime} \rightarrow 0
$$

where $M^{\prime}$ and $M^{\prime \prime}$ are in $\mathcal{D}$. Since $\mathcal{D}$ is closed under kernels and cokernels, $0 \in \mathcal{D}$ (unless $\mathcal{D}$ is empty), so that $\mathcal{D}_{1} \supseteq \mathcal{D}$.

We claim that $\mathcal{D}_{1}$ is still closed under kernels and cokernels. We will prove this after the following lemma.

Lemma 4.2. Suppose $\mathcal{A}$ is an abelian category and the full subcategory $\mathcal{D}$ of $\mathcal{A}$ is closed under kernels and cokernels. Let $\mathcal{D}_{1}$ be the full subcategory consisting of extensions of $\mathcal{D}$. Suppose $M \in \mathcal{D}, N \in \mathcal{D}_{1}$, and $f: M \rightarrow N$ is a map. Then $\operatorname{ker} f \in \mathcal{D}$ and $\operatorname{cok} f \in \mathcal{D}_{1}$.

Proof. Because $N \in \mathcal{D}_{1}$, we can construct the commutative diagram

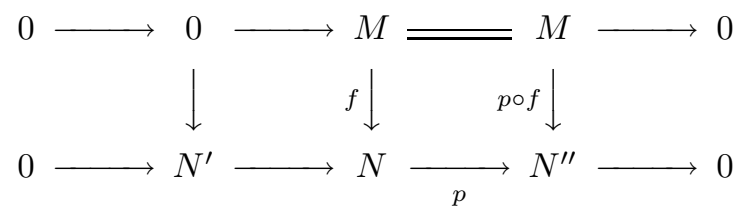


where the rows are exact and $N^{\prime}, N^{\prime \prime} \in \mathcal{D}$. The snake lemma then gives us an exact sequence

$$
0 \rightarrow \operatorname{ker} f \rightarrow \operatorname{ker}(p \circ f) \stackrel{\partial}{\rightarrow} N^{\prime} \rightarrow \operatorname{cok} f \rightarrow \operatorname{cok}(p \circ f) \rightarrow 0 .
$$

Since $\operatorname{ker}(p \circ f)$ and $N^{\prime}$ are in $\mathcal{D}$, we find that $\operatorname{ker} f=\operatorname{ker} \partial$ is in $\mathcal{D}$. Similary, we find that $\operatorname{cok} f$ is an extension of $\operatorname{cok} \partial$ and $\operatorname{cok}(p \circ f)$, so cok $f \in \mathcal{D}_{1}$.

Proposition 4.3. Suppose $\mathcal{A}$ is an abelian category, and $\mathcal{D}$ is a full subcategory of $\mathcal{A}$ closed under kernels and cokernels. Let $\mathcal{D}_{1}$ be the full subcategory consisting of extensions of $\mathcal{D}$. Then $\mathcal{D}_{1}$ is also closed under kernels and cokernels.

Proof. Suppose $f: M \rightarrow N$ is a map of $\mathcal{D}_{1}$. Then we have the commutative diagram

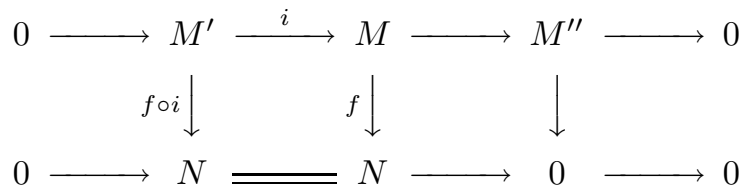

where the rows are exact and $M^{\prime}, M^{\prime \prime} \in \mathcal{D}$. The snake lemma gives an exact sequence

$$
0 \rightarrow \operatorname{ker}(f \circ i) \rightarrow \operatorname{ker} f \rightarrow M^{\prime \prime} \stackrel{\partial}{\rightarrow} \operatorname{cok}(f \circ i) \rightarrow \operatorname{cok} f \rightarrow 0 .
$$

Hence $\operatorname{ker} f$ is an extension of $\operatorname{ker}(f \circ i)$ and $\operatorname{ker} \partial$, both of which are in $\mathcal{D}$ by Lemma 4.2 So $\operatorname{ker} f \in \mathcal{D}_{1}$. Similarly, $\operatorname{cok} f=\operatorname{cok} \partial$, which is in $\mathcal{D}_{1}$ by Lemma 4.2

Corollary 4.4. Suppose $\mathcal{A}$ is an abelian category, and $\mathcal{D}$ is a full subcategory of $\mathcal{A}$ closed under kernels and cokernels. Let $\mathcal{D}_{0}=\mathcal{D}$, and, for $n \geq 1$, define $\mathcal{D}_{n}$ to be the full subcategory of extensions of $\mathcal{D}_{n-1}$. Let $\mathcal{E}=\bigcup_{n=0}^{\infty} \mathcal{D}_{n}$. Then $\mathcal{E}$ is the wide subcategory generated by $\mathcal{D}$.

Proof. Note that the union that defines $\mathcal{E}$ is an increasing one, in the sense that $\mathcal{D}_{n} \subseteq \mathcal{D}_{n+1}$. This makes it clear that $\mathcal{E}$ is closed under extensions. Proposition 4.3 implies that $\mathcal{E}$ is closed under kernels and cokernels. Therefore, $\mathcal{E}$ is a wide subcategory. Since any wide subcategory containing $\mathcal{D}$ must contain each $\mathcal{D}_{n}$, the corollary follows.

Theorem 4.5. Suppose $R$ is a coherent ring, and $\mathfrak{a}$ is a two-sided ideal of $R$ that is finitely generated as a left ideal. Then the map $u: \mathcal{L}_{\text {wide }}(R / \mathfrak{a}) \rightarrow \mathcal{L}_{\text {wide }}(R)$ is an embedding.

Proof. It suffices to show that $g f(\mathcal{D})=\mathcal{D}$, where $\mathcal{D}$ is a wide subcategory of $\mathcal{C}_{0}(R / \mathfrak{a})$. According to Corollary 4.4, $u(\mathcal{D})=\bigcup_{n} \mathcal{D}_{n}$, where $\mathcal{D}_{n}$ is the collection of extensions of $\mathcal{D}_{n-1}$. We prove by induction on $n$ that if $\mathfrak{a}$ acts trivially on some $M \in \mathcal{D}_{n}$, then in fact $M \in \mathcal{D}$. The base case of the induction is clear, since $\mathcal{D}_{0}=\mathcal{D}$. Now suppose our claim is true for $n-1$, and $\mathfrak{a}$ acts trivially on $M \in \mathcal{D}_{n}$. Write $M$ as an extension

$$
0 \rightarrow M^{\prime} \rightarrow M \rightarrow M^{\prime \prime} \rightarrow 0
$$

where $M^{\prime}, M^{\prime \prime} \in \mathcal{D}_{n-1}$. Then $\mathfrak{a}$ acts trivially on $M^{\prime}$ and $M^{\prime \prime}$, so $M^{\prime}, M^{\prime \prime} \in \mathcal{D}$. Furthermore, this is an extension of $R / \mathfrak{a}$-modules; since $\mathcal{D}$ is a wide subcategory of $R / \mathfrak{a}$-modules, $M \in \mathcal{D}$. The induction is complete, and we find that $g f(\mathcal{D})=\mathcal{D}$. 
Theorem 4.6. Suppose $R$ is a commutative coherent ring such that

$$
f_{R}: \mathcal{L}_{\text {wide }}(R) \rightarrow \mathcal{L}_{\text {thick }}(\mathcal{D}(R))
$$

is an isomorphism, and $\mathfrak{a}$ is a finitely generated ideal of $R$. Then $f_{R / \mathfrak{a}}$ is also an isomorphism. In particular, $f_{R}$ is an isomorphism for all rings $R$ that are quotients of regular commutative coherent rings by finitely generated ideals.

Proof. The second statement follows immediately from the first and Theorem 3.6 To prove the first statement, note that, by hypothesis and Thomason's Theorem 2.1 the map

$$
G_{R}=\alpha j i: \widetilde{J}(\operatorname{Spec} R) \rightarrow \mathcal{L}_{\text {wide }}(R)
$$

is an isomorphism. It suffices to show that $G_{R / \mathfrak{a}}$ is a surjection. So suppose $\mathcal{D}$ is a wide subcategory of finitely presented $R / \mathfrak{a}$-modules. Then $u \mathcal{D}=G(S)$ for some $S \in \widetilde{J}(\operatorname{Spec} R)$. Since $M_{(\mathfrak{p})}=0$ for any $R$-module $M$ such that $\mathfrak{a} M=0$ and any $\mathfrak{p}$ not containing $\mathfrak{a}$, we have $S=T \cup D(\mathfrak{a})$ for a unique $T \subseteq V(\mathfrak{a})=\operatorname{Spec}(R / \mathfrak{a})$. One can easily see that $T \in J(\operatorname{Spec} R / \mathfrak{a})$, but we claim that in fact $T \in \widetilde{J}(\operatorname{Spec} R / \mathfrak{a})$. Indeed, $T=S \cap V(\mathfrak{a})$, so this claim boils down to showing that the inclusion $V(\mathfrak{a}) \subseteq \operatorname{Spec} R$ is a proper map. This is well-known; the inclusion of any closed subset in any topological space is proper.

Naturally, we claim that $G_{R / \mathfrak{a}}(T)=\mathcal{D}$. We have $\mathcal{D} \subseteq u \mathcal{D}=G(S) \subseteq G(T)$, since $S \supseteq T$. To show the converse, it suffices to show that $u G_{R / \mathfrak{a}}(T) \subseteq u \mathcal{D}=G(S)$, since $u$ is an embedding by Theorem 4.5. But any module $M$ in $G_{R / \mathfrak{a}}(T)$ has $M_{(\mathfrak{p})}=0$ for all $\mathfrak{p} \in T$ and for all $\mathfrak{p} \in D(\mathfrak{a})$. Therefore, $u G_{R / \mathfrak{a}}(T) \subseteq G(T \cup D(\mathfrak{a}))=G(S)$.

Corollary 4.7. Suppose $R$ is a finitely generated commutative $k$-algebra, where $k$ is a principal ideal domain. Then every wide subcategory of finitely generated $R$-modules is a Serre class.

Indeed, any such $R$ is a quotient of a finitely generated polynomial ring over $k$, which is regular by Hilbert's syzygy theorem, by a finitely generated ideal. This corollary covers most of the Noetherian rings in common use, though of course it does not cover all of them. We remain convinced that $f$ should be an isomorphism for all commutative coherent rings.

\section{LOCALIZING SUBCATEGORIES}

In this section, we use Neeman's classification of localizing subcategories (thick subcategories closed under direct sums) of $\mathcal{D}(R)$ for Noetherian commutative $R$ to deduce a classification of wide subcategories of $R$-modules closed under direct sums. We know of no direct proof of this classification.

Let $\mathcal{L}_{\text {wide }}^{\oplus}(R)$ denote the lattice of wide subcategories of $R$-modules closed under arbitrary coproducts, and let $\mathcal{L}_{\text {thick }}^{\oplus}(\mathcal{D}(R))$ denote the lattice of localizing subcategories of $\mathcal{D}(R)$. Just as before, we can define a map $f: \mathcal{L}_{\text {wide }}^{\oplus}(R) \rightarrow \mathcal{L}_{\text {thick }}^{\oplus}(R)$, where $f(\mathcal{C})$ is the collection of all $X$ such that $H_{n} X \in \mathcal{C}$ for all $n$. The proof of Proposition 1.2 goes through without difficulty to show that $f(\mathcal{C})$ is localizing.

Similarly, we can define $g: \mathcal{L}_{\text {thick }}^{\oplus}(\mathcal{D}(R)) \rightarrow \mathcal{L}_{\text {wide }}^{\oplus}(R)$ by letting $g(\mathcal{D})$ be the smallest wide subcategory closed under coproducts containing all the $H_{n} X$, for $X \in \mathcal{D}$ and for all $n$. The proof of Proposition 1.4 goes through without change, showing that $g$ is left adjoint to $f$. 
Lemma 5.1. For any ring $R$ and any wide subcategory $\mathcal{C}$ of $R$-modules closed under coproducts, we have $g f(\mathcal{C})=\mathcal{C}$.

Proof. Since $g$ is left adjoint to $f, g f(\mathcal{C}) \subseteq \mathcal{C}$. But, given $M \in \mathcal{C}, S^{0} M$ is in $f(\mathcal{C})$, and hence $M=H_{0} S^{0} M \in g f(\mathcal{C})$.

It would be surprising if an arbitrary localizing subcategory of $\mathcal{D}(R)$ were determined by the homology groups of objects in it, but this is nevertheless the case when $R$ is Noetherian and commutative. Given a prime ideal $\mathfrak{p}$ of such an $R$, denote by $k_{\mathfrak{p}}$ the residue field $R_{(\mathfrak{p})} / \mathfrak{p}$ of $\mathfrak{p}$.

Theorem 5.2. Suppose $R$ is a Noetherian commutative ring. Then $f: \mathcal{L}_{\text {wide }}^{\oplus}(R) \rightarrow$ $\mathcal{L}_{\text {thick }}^{\oplus}(\mathcal{D}(R))$ is an isomorphism. Furthermore, there is an isomorphism between the Boolean algebra $2^{\operatorname{Spec} R}$ and $\mathcal{L}_{\text {wide }}^{\oplus}(R)$ that takes a set $A$ of prime ideals to the wide subcategory closed under coproducts generated by the $k_{\mathfrak{p}}$ for $\mathfrak{p} \in A$.

Proof. We have a map $\alpha: 2^{\operatorname{Spec} R} \rightarrow \mathcal{L}_{\text {wide }}^{\oplus}(R)$, defined as in the statement of the theorem. The composition $f \alpha$ is proved to be an isomorphism, for $R$ Noetherian and commutative, in [Nee92] (see also [HPS97. Sections 6 and 9]). Since $f$ is injective, we conclude that $f$, and hence also $\alpha$, is an isomorphism.

For example, the wide subcategory closed under coproducts of abelian groups corresponding to the prime ideal 0 is the collection of rational vector spaces; the wide subcategory closed under coproducts corresponding to the set $\{0, p\}$ is the collection of $p$-local abelian groups.

\section{REFERENCES}

[CKN99] J. Daniel Christensen, Bernhard Keller, and Amnon Neeman, Failure of Brown representability in derived categories, preprint, 1999.

[Gla89] Sarah Glaz, Commutative coherent rings, Lecture Notes in Mathematics no. 1371, Springer-Verlag, Berlin, 1989. MR 90f:13001

[Har77] Robin H. Hartshorne, Algebraic geometry, Graduate Texts in Mathematics, vol. 52, Springer-Verlag, 1977. MR 57:3116

[Hop87] Michael J. Hopkins, Global methods in homotopy theory, Homotopy theory (Durham, 1985) (J. D. S. Jones and E. Rees, eds.), London Math. Soc. Lecture Note Ser., vol. 117, Cambridge Univ. Press, Cambridge-New York, 1987, pp. 73-96. MR 89g:55022

[Hov98] Mark Hovey, Model categories, Mathematical Surveys and Monographs, vol. 63, American Mathematical Society, Providence, RI, 1999. MR 99h:55031

[HP99] Mark Hovey and John H. Palmieri, Stably thick subcategories of modules over Hopf algebras, to appear in Math. Proc. Camb. Phil. Soc.

[HP00] Mark Hovey and John H. Palmieri, Galois theory of thick subcategories in modular representation theory, J. Algebra 230 (2000), 713-729. CMP 2000:16

[HPS97] Mark Hovey, John H. Palmieri, and Neil P. Strickland, Axiomatic stable homotopy theory, Mem. Amer. Math. Soc. 128 (1997), no. 610, x+114. MR 98a:55017

[HS98] Michael J. Hopkins and Jeffrey H. Smith, Nilpotence and stable homotopy theory. II, Ann. of Math. (2) 148 (1998), no. 1, 1-49. MR 99h:55009

[Nee92] A. Neeman, The chromatic tower for $D(R)$, Topology 31 (1992), 519-532. MR 93h:18018

[Ste75] B. Stenström, Rings of quotients, Die Grundlehren der mathematischen Wissenschaften, vol. 217, Springer-Verlag, Berlin, 1975. MR 52:10782

[Tho97] R. W. Thomason, The classification of triangulated subcategories, Compositio Math. 105 (1997), no. 1, 1-27. MR 98b:18017

Department of Mathematics, Wesleyan University, Middletown, Connecticut 06459

E-mail address: hovey@member.ams.org 